http://jmscr.igmpublication.org/home/ ISSN (e)-2347-176x ISSN (p) 2455-0450 crossref DOI: https://dx.doi.org/10.18535/jmscr/v9i3.16

\title{
Prevalence of Tumor Lysis Syndrome in Acute Leukemia and Lymphoreticular Malignancies: A Regional Study from PGIMS, Rohtak
}

\author{
Authors \\ Sudhir Kumar Atri ${ }^{1}$, Ekta Devi ${ }^{2}$, Homdutt $^{3^{*}}$, Shiv Kumar ${ }^{4}$, Bhanu Pratap Singh ${ }^{5}$ \\ ${ }^{1}$ Senior Professor, Department of Medicine, Pt. B.D. Sharma, PGIMS, Rohtak \\ ${ }^{2}$ Senior Resident, Department of Medicine, Pt. B.D.Sharma, PGIMS, Rohtak \\ 3,4,5 Junior Resident, Department of Medicine, Pt. B.D.Sharma, PGIMS, Rohtak \\ *Corresponding Author \\ Homdutt
}

Junior Resident, Department of Medicine, Pt. B.D. Sharma PGIMS, Rohtak, Haryana - 124001, India

\begin{abstract}
Objectives: This study was conducted to determine the prevalence of tumor lysis syndrome (TLS) in patients of acute leukemia and lymphoreticular malignancies (Lymphoma).

Materials and Methods: The present study was conducted at Pandit Bhagwat Dayal Sharma Post Graduate Institute of Medical Sciences, Rohtak and data was collected bynon-probability convenient sampling from hundred patients (age 14 years or above) of Acute Leukemia or Lymphoma in Haematology Department of Medicine over a period of one year. Patients satisfying inclusion criteria were monitored for development of tumor lysis syndrome. Starting from day of admission till completion of 4 weeks of induction therapy in leukemia patients following parameters (Serum Uric Acid, Serum Potassium, Serum Phosphorus, Serum Calcium, Blasts and TLC) were monitored on alternate day to see for development of TLS. Starting from day of admission, monitoring of same parameters was done on day 1 and day 2 of each cycle of lymphoma patients for TLS. If Tumor Lysis Syndrome developed (Grade II or more) then monitoring was done on each day till remission. All patients received adequate hydration, allopurinol and induction chemotherapy. Data were analysed by statistical package for social sciences (SPSS) version16.0.

Results: Out of 100 patients 19 fulfilled the criteria for TLS.In acute leukemia group 10 (28.57\%) patients developed TLS of which 5 patients $(14.3 \%)$ had spontaneous tumor lysis syndrome, 4 patients $(11.4 \%)$ had laboratory tumor lysis syndrome and only one patient (2.9\%) had clinical tumor lysis syndrome. Among lymphoma group no patient developed spontaneous or clinical tumor lysis syndrome. Only laboratory tumor lysis syndrome was seen in 9 (13.8\%) out of 65 patients of lymphoma. Overall one patient died because of TLS. Hyperuricemia and Hyperkalemia were the most prominent findings in patients with tumor lysis syndrome.

Conclusion: It is concluded that 19\% of the patients developed TLS (including both laboratory and clinical TLS) and despite all measures of prevention it can occur and result in devastating clinical effects.

Keywords: Tumor lysis syndrome, hyperuricemia, hyperphosphatemia, hypocalcaemia, acute renal failure.
\end{abstract}




\section{Introduction}

The tumor lysis syndrome is the most common disease-related emergency encountered by physicians caring for patients with hematologic cancers. ${ }^{1}$ Although it develops most often in patients with non-Hodgkin's lymphoma or acute leukemia, its frequency is increasing among patients who have tumors that used to be only rarely associated with this complication. ${ }^{2,3}$ The tumor lysis syndrome occurs when tumor cells release their contents into the bloodstream, either spontaneously or in response to therapy, leading to the characteristic findings of hyperuricemia, hyperkalemia, hyperphosphatemia, and hypocalcemia. ${ }^{1}$ These electrolyte and metabolic disturbances can progress to clinical toxic effects, including renal insufficiency, cardiac arrhythmias, seizures, and death due to multi organ failure. Thus TLS is an oncological emergency triggered by rapid release of intracellular material from lysing malignant cells by radiotherapy, chemotherapy or spontaneously. The clinically relevant definition of TLS was developed by Cairo and Bishop.

\section{Definition of Laboratory Tumour Lysis Syndrome (LTLS) ${ }^{4}$}

- Uric acid $\geq 476 \mathrm{mmol} / 1$ or $>8 \mathrm{mg} / \mathrm{dl}$ or $25 \%$ increase from baseline

- Potassium $\geq 60 \mathrm{mmol} / 1$ or $>6 \mathrm{mEq} / \mathrm{L}$ or $25 \%$ increase from baseline

- Phosphate $\geq 145 \mathrm{mmol} / 1$ or $>4.5 \mathrm{mg} / \mathrm{dL}$ or $25 \%$ increase from baseline

- Calcium $\leq 175 \mathrm{mmol} / 1$ or $<7 \mathrm{mg} / \mathrm{dL}$ or $25 \%$ decrease from baseline

Laboratory tumorlysis syndrome (LTLS) is defined as either a $25 \%$ change of level above or below normal, as defined above, for any two or more serum values of given biochemical markers within 3 days before or 7 days after the initiation of chemotherapy.
Definition of Clinical Tumour Lysis Syndrome $(\text { CTLS })^{4}$

1. Creatinine more than 1.5 times upper limit of normal (age adjusted value)

2. Cardiac arrhythmia/sudden death

3. Seizure

Clinical tumour lysis syndrome is labelled once there is LTLS in addition to one or more of the abovementioned criteria.

\section{Materials and Methods}

Patients: This descriptive observational study was conducted at Pandit Bhagwat Dayal Sharma Post Graduate Institute of Medical Sciences, Rohtak. One hundred patients presenting with tissue diagnosis of acute leukemia (based on bone marrow aspiration, biopsy and immunophenotyping) and lymphoma (based on biopsy and immune-histochemistry) were included in the study.

\section{Inclusion Criteria}

- Age should be more than 14 years

- Diagnosed case of leukemia(on basis of presence of $>20 \%$ blasts either on peripheral blood or bone marrow by Immuno-phenotyping or flow cytometry) and lymphoma (biopsy and Immunohistochemistry markers proven).

- Receiving first course of chemotherapy with minimum GFR of $90 \mathrm{ml} / \mathrm{min} / \mathrm{m} 2$.

\section{Exclusion Criteria}

- Patient receiving uricosuric agents for indication other than TLS

○ Post renal transplant

During the twelve month study period 105 cases of Acute leukemia and lymphoma presented to the ward but 5 of them were not selected for study because 3 patients already had received chemotherapy at some other hospital and 2 were on uricosuric agents. Therefore total 100 patients were included in the study. After obtaining the informed consent, clinical history was taken and physical examination was performed. Baseline electrocardiography of each patient was recorded 
at presentation and was repeated if patient developed any symptoms or noticed to have arrhythmia. Venous blood samples were drawn in EDTA tube for total leucocyte count (TLC) and were analyzed on Sysmexanalyzer. Venous blood samples were drawn to determine the levels of serum phosphate, potassium, uric acid, creatinine, calcium at presentation and then were checked on alternate day from the day before starting chemotherapy (day 0) up to day 28 in patients of leukemia and on day 1 and day 2 of each cycle in patients of lymphoma for development of TLS. In cases who developed TLS monitoring was done on each day till remission. All patients received hydration and allopurinol therapy once they got admitted and were started with induction chemotherapy. All patients withAcute lymphoblastic leukemia received chemotherapy including vincristine, adriamycin, asparginase and prednisone (multiagent induction). All patients with Acute myeloid leukemia received daunorubicin, cytrabine and arabinoside $(3+7$ induction) except patients with Acute promyelocytic leukemia (APML) who were offered All-Trans-Retinoic-Acid (ATRA) with daunorubicin. All patients with Non Hodgkin's
Lymphoma received CHOP regimen (including cyclophosphamide, adriamycin, vincristine and prednisone) for B cell type and CHOEP (CHOP + Etoposide) for $\mathrm{T}$ cell type. Patients with Hodgkin's disease (HD) received ABVD (adriamycin, bleomycin, vinblastine and dacarbazine). Data were analysed by statistical package for social sciences (SPSS) version 16.0 and relevant descriptive statistics were calculated. Frequency and percentages were computed for presentation of qualitative response variables of the study e.g. TLS, LTLS and CTLS. Quantitative variables like serum uric acid, calcium, potassium, phosphate, Creatinine were presented with mean and Standard Deviation. Independent sample ttest was used to compare mean difference between patients with TLS and those without TLS for quantitative variables like TLC, serum uric acid, calcium, potassium, phosphate, Creatinine. $\mathrm{P}$ value 0.05 or less was considered as significant.

\section{Statistical Analysis}

At the end of study data was collected, compiled and analysed using appropriate statistical tests and prevalence of Tumor Lysis Syndrome was calculated.

\section{Results}

\section{Patient Characteristics}

Table 1: Base line Biochemical Parameters in the Patients

\begin{tabular}{|l|c|c|}
\hline Parameters & Values :LEUKEMIA & Values: LYMPHOMA \\
\hline Blood urea $(\mathrm{mg} \%)$ & $26.89 \pm 10.90$ & $26.14 \pm 8.07$ \\
\hline Serum Creatinine $(\mathrm{mg} \%)$ & $0.74 \pm 0.22$ & $0.91 \pm 0.78$ \\
\hline Random Blood Sugar $(\mathrm{mg} \%)$ & $100.51 \pm 11.19$ & $100.85 \pm 4.32$ \\
\hline Sodium(meq/l) & $140.06 \pm 4.14$ & $141.55 \pm 3.23$ \\
\hline Potassium(meq/l) & $4.19 \pm 0.61$ & $4.01 \pm 0.42$ \\
\hline Serum calciu m(mg/dl) & $8.83 \pm 0.88$ & $8.83 \pm 0.77$ \\
\hline Serum phosphate(mg/dl) & $3.32 \pm 1.00$ & $3.19 \pm 0.65$ \\
\hline Serum urate( $\mathrm{mg} / \mathrm{dl})$ & $4.84 \pm 2.26$ & $4.81 \pm 1.11$ \\
\hline Seru m protein $(\mathrm{g} / \mathrm{dl})$ & $6.85 \pm 0.99$ & $7.48 \pm 0.81$ \\
\hline A/G ratio & $1.24 \pm 0.55$ & $1.10 \pm 0.18$ \\
\hline Haemoglobin $(\mathrm{g} \mathrm{m} \%)$ & $7.74 \pm 2.50$ & $10.31 \pm 2.38$ \\
\hline Platelet count $(\mathrm{lac})$ & $0.45 \pm 0.63$ & $2.20 \pm 1.29$ \\
\hline Total Leucocyte count $\left(\mathrm{cells} / \mathrm{mm}^{3}\right)$ & $119308.47 \pm 500$ & $10407.85 \pm 7403.92$ \\
\hline Blasts $(\%)$ & $45.34 \pm 33.13$ & - \\
\hline
\end{tabular}

Around half of acute leukemia patients presented in young age of 15-25 years. In lymphoma patients no such age predominance is seen.
Among total 35 leukemia patients nearly half (54.3\%) were diagnosed as acute lymphocytic leukemia and rest were of acute myeloid 
leukemia. Out of 19 patients of ALL 18 were of B cell ALL and only one patient hadT- ALL. In Lymphoma group out of 65 patients 35 patients were of NHL and rest 30 patients were of Hodgkin's Lymphoma. Of total 65 patients $26.2 \%$ were of follicular lymphoma $20 \%$ each of Mixed cellularity and Nodular sclerosis type of HL, $13.8 \%$ of DLBCL, $9.2 \%$ peripheral $\mathrm{T}$ cell NHL,
4.6\% Non lymphocytic predominant Hodgkin's Lymphoma, 3.1\% Small lymphocytic lymphoma and $1.5 \%$ of each lymphocytic rich and spleenic marginal zone lymphoma. Nearly $21 \%$ cases presented with early disease i.e. up to stage II A, $4.6 \%$ with stage II B and rest $73.9 \%$ with advanced disease including stage III and IV.

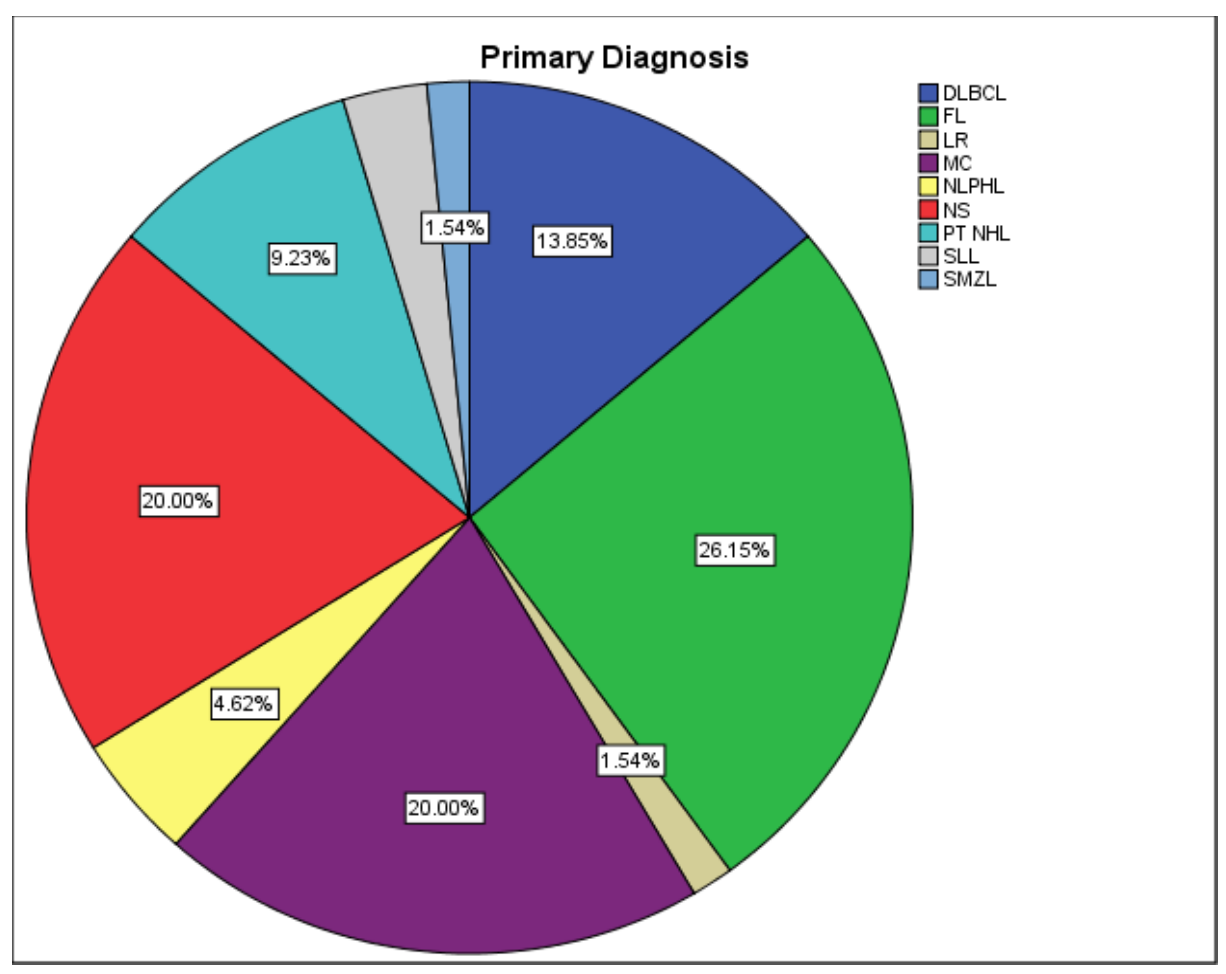

Pie chart showing Primary diagnosis in lymphoma patients

In patients of acute leukemia, only two patients had past history of associated co-morbidity in form of COPD and Diabetes mellitus. Among lymphoma group 3 patients had history of old treated pulmonary TB, 2 patients had COPD and 1 patient was hypertensive.

Nearly $60 \%$ of leukemia patients presented with fever, $22.9 \%$ with fatigue, $8.6 \%$ with mass abdomen and rest $8.6 \%$ with bleeding manifestation in form of petechial spots $(5.7 \%)$ or bleeding per vagina (2.9\%). Majority of lymphoma patients presented with either lymphadenopathy (49.2\%) or fever $(27.7 \%)$. Few patients presented with only left hypochondriac pain $(4.6 \%)$ or abdominal mass $(3.1 \%)$ or nasal mass $(1.5 \%)$ or scrotal swelling $(1.5 \%)$.Pallor was present in nearly $97 \%$ patients of leukemia and only $44.6 \%$ of lymphoma patients at the time of presentation. Icterus was not present in any leukemia case and was seen only in two patients of lymphoma. Spleenomegaly was present in nearly $45 \%$ cases of leukemia and only $21 \%$ cases of lymphoma. Out of 35 patients of leukemia only $14(40 \%)$ patients had lymphadenopathy while majority $(90.8 \%)$ of lymphoma cases presented with lymphadenopathy

Among 30 patients of acute leukemia, all patients of B ALL were given augmented BFM regimen and patients of acute myeloid leukemia were given either $3+7(22.9 \%)$ or Azacytidine $(8.6 \%)$ or Decitabine $(14.3 \%)$ as induction chemotherapy depending on age and hemodynamic condition of patients. In Lymphoma group individualized chemotherapy based on type of lymphoma was 
given : 27 patients were given chemotherapy as ABVD regimen, 20 patients as $\mathrm{R}$ CHOP, 8 patients as CHOP, 5 patients as CHOP E, 2 patients as RABVD and 1 patient as RDHAP. In our study in acute leukemia group 10 (28.57\%) out of 35 patients developed tumor lysis syndrome of which 5 patients (14.3\%) had spontaneous tumor lysis syndrome, 4 patients (11.4\%) had laboratory tumor lysis syndrome and only one patient $(2.9 \%)$ had clinical tumor lysis syndrome

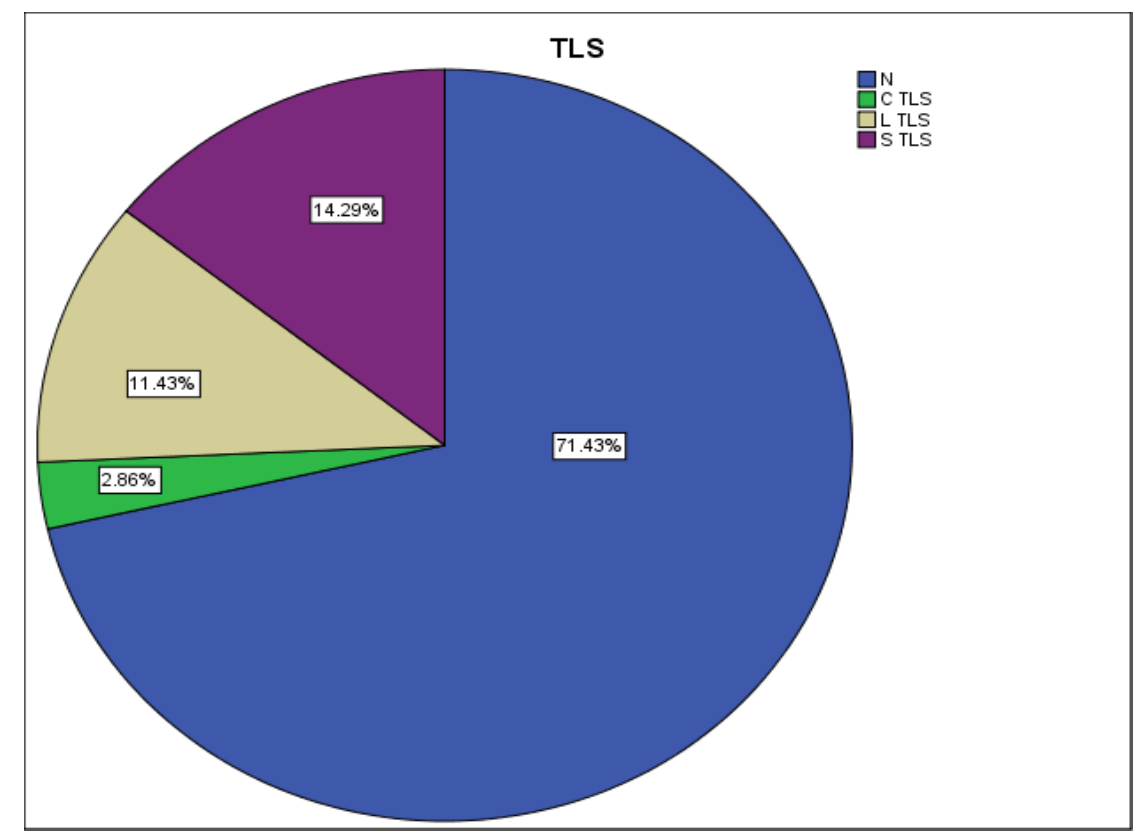

Pie chart showing prevalence of TLS in acute leukemia patients

Among lymphoma group no patient developed spontaneous or clinical tumor lysis syndrome. Only laboratory tumor lysis syndrome was seen in $9(13.8 \%)$ out of 65 patients of lymphoma. Among acute leukemia TLS patients $80 \%$ were of age 15 to 25 years and rest $20 \%$ of age 36- 45 years. In lymphoma group out of 9 patients who developed TLS $33.3 \%$ were of age $>55$ years and age between 36-45 years each, $22.2 \%$ were of age $15-25$ years and rest $11.1 \%$ of age $26-35$ years.In acute leukemia group, 8 out of 10 patients who developed TLS were of B ALL amounting for nearly $80 \%$ of cases. Only one patient of acute myeloid leukemia and T ALL each developed tumor lysis syndrome. In Lymphoma group all 9 patients who developed TLS were having advanced disease. 4 patients $(44.4 \%)$ were of stage III B, 3 patients (33.3\%) of stage IV B and 2 patients $(22.2 \%)$ of stage IV BX. All patients who presented with stage IV BX developed tumor lysis syndrome.Among Lymphoma group, out of 9 patients who developed TLS 5 patients (55.55\%) were of Hodgkin's Lymphoma including 4 patients of mixed cellularity and one patient of nodular lymphocytic predominant type of Hodgkin's Lymphoma and rest 4 patients (44.44\%) were of Non Hodgkin's Lymphoma including 3 patients of follicular lymphoma and one patient of DLBCL.

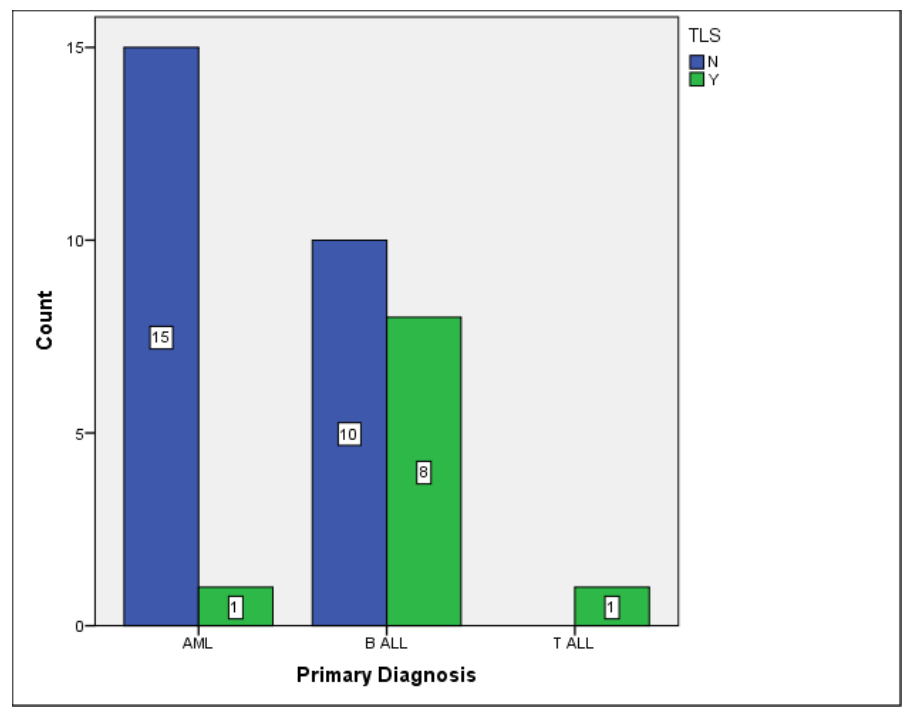

Graph showing Diagnosis based distribution of TLS among leukemia patients. 


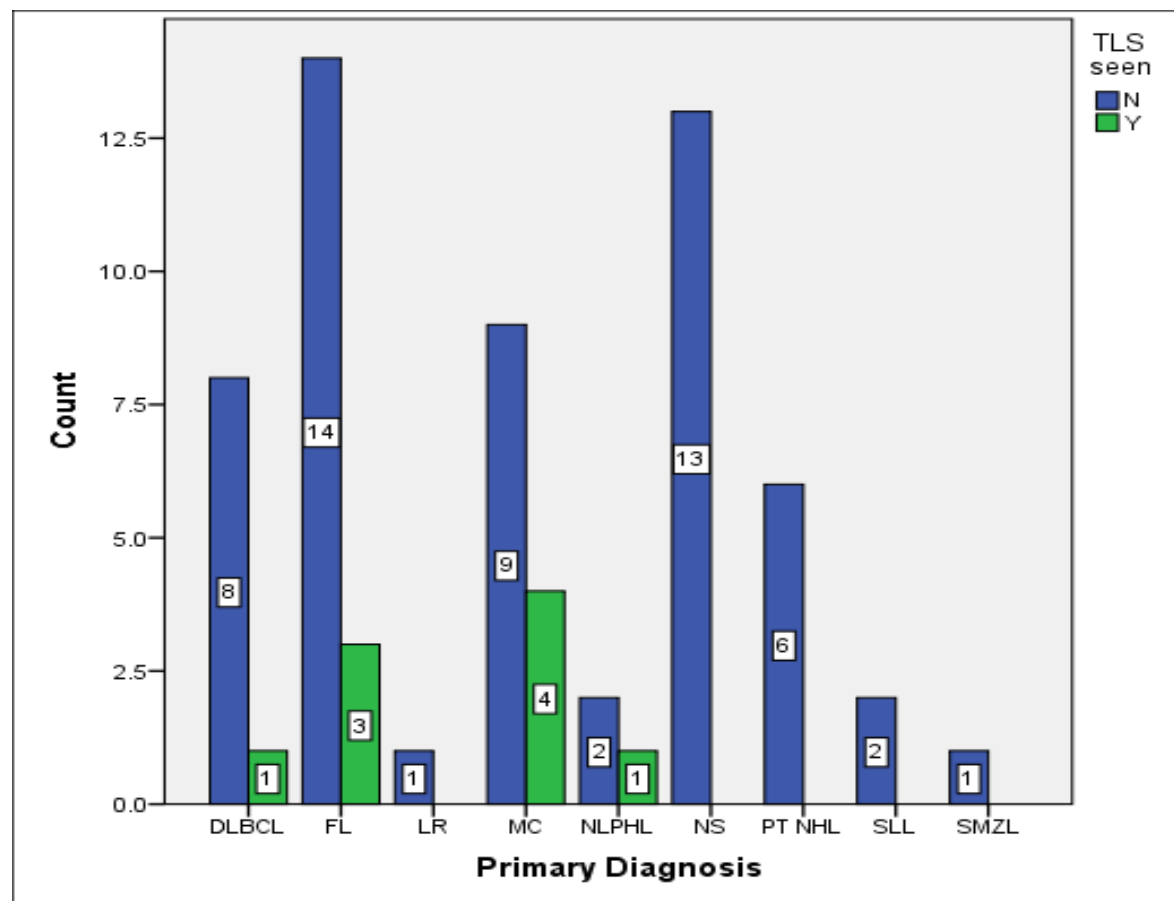

Graph s howing Diagnosis based distribution of TLS among lymphoma patients.

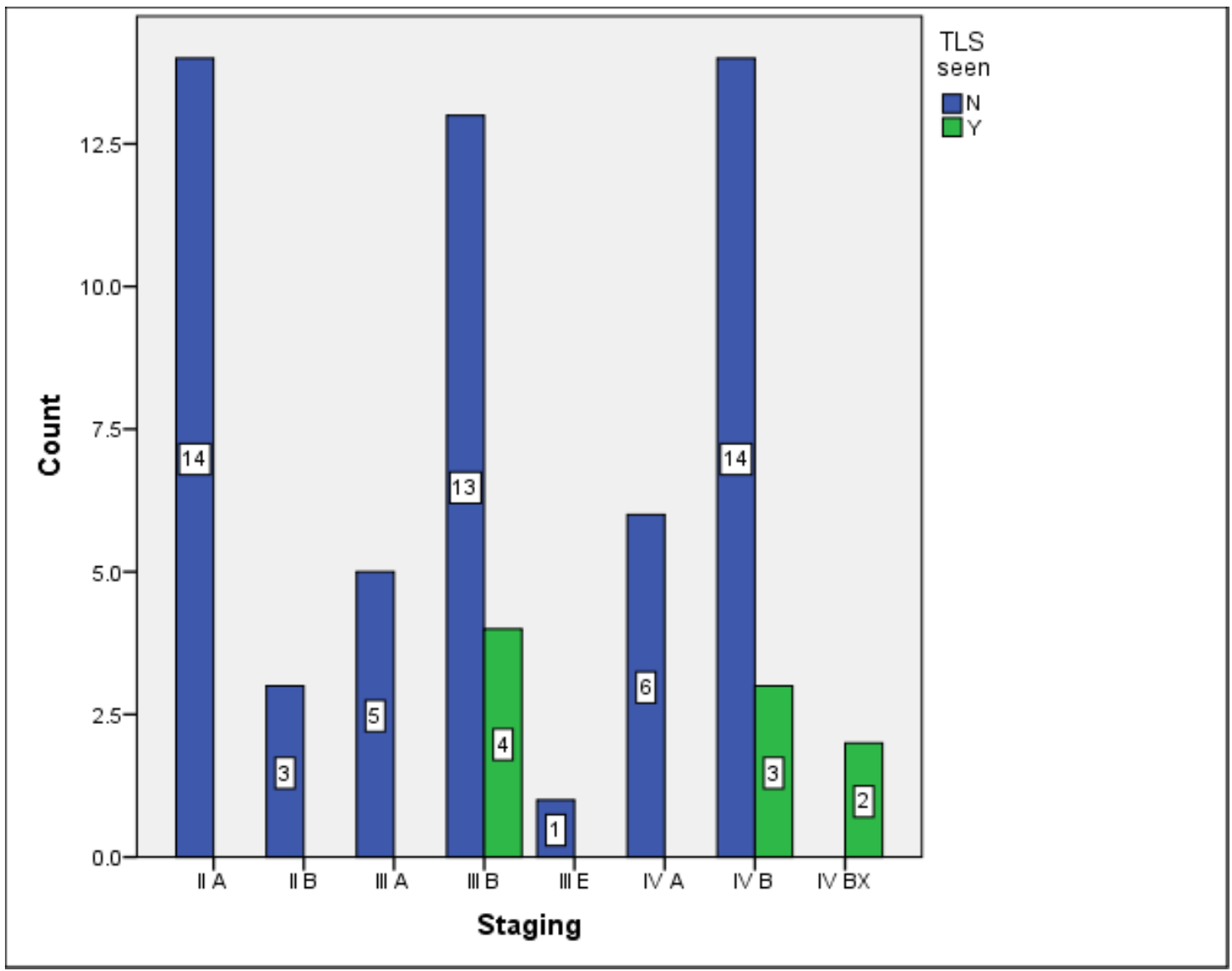

Graph showing Stage wise distribution of TLS among lymphoma patients.

9 out of 10 patients of acute leukemia who developed TLS were on augmented BFM regimen. In Lymphoma group out of 9 patients who developed TLS 4 patients were on ABVD, 3 patients on R CHOP, 1 patient each on R DHAP and CHOP based chemotherapy 


\begin{tabular}{|c|c|c|c|c|c|}
\hline & TLS & $\mathrm{N}$ & Mean & Std. Deviation & $P$ value \\
\hline \multirow{2}{*}{ Age } & $\mathrm{Y}$ & 10 & 21.40 & 9.629 & \\
\hline & $\mathrm{N}$ & 25 & 32.08 & 17.054 & .027 \\
\hline \multirow{2}{*}{ BMI } & $\mathrm{Y}$ & 10 & 23.4494 & 3.69129 & .677 \\
\hline & $\mathrm{N}$ & 25 & 22.8260 & 4.05411 & \\
\hline \multirow{2}{*}{ Pulse rate } & $\mathrm{Y}$ & 10 & 85.60 & 3.978 & .242 \\
\hline & $\mathrm{N}$ & 25 & 83.16 & 5.942 & \\
\hline \multirow{2}{*}{ SBP } & $\mathrm{Y}$ & 10 & 108.40 & 7.043 & .094 \\
\hline & $\mathrm{N}$ & 25 & 113.84 & 8.887 & \\
\hline \multirow{2}{*}{ DBP } & $\mathrm{Y}$ & 10 & 69.60 & 5.948 & .140 \\
\hline & $\mathrm{N}$ & 25 & 73.44 & 7.083 & \\
\hline \multirow{2}{*}{ B Urea } & $\mathrm{Y}$ & 10 & 37.00 & 9.274 & .001 \\
\hline & $\mathrm{N}$ & 25 & 22.84 & 8.740 & \\
\hline \multirow{2}{*}{ S Creatinine } & $\mathrm{Y}$ & 10 & .820 & .2658 & .217 \\
\hline & $\mathrm{N}$ & 25 & .716 & .2014 & . \\
\hline \multirow{2}{*}{ S Sodium } & $\overline{\mathrm{Y}}$ & 10 & 140.10 & 4.818 & .970 \\
\hline & $\mathrm{N}$ & 25 & 140.04 & 3.953 & \\
\hline \multirow{2}{*}{ S Potassium } & $\mathrm{Y}$ & 10 & 4.520 & .9271 & .166 \\
\hline & $\mathrm{N}$ & 25 & 4.068 & .3902 & \\
\hline \multirow{2}{*}{ S Calcium } & $\mathrm{Y}$ & 10 & 8.370 & 1.0636 & .048 \\
\hline & $\overline{\mathrm{N}}$ & 25 & 9.020 & .7450 & \\
\hline \multirow{2}{*}{ S Phosphate } & $\mathrm{Y}$ & 10 & 3.430 & 1.1519 & .711 \\
\hline & $\mathrm{N}$ & 25 & 3.288 & .9619 & \\
\hline \multirow{2}{*}{ S Uric acid } & $\mathrm{Y}$ & 10 & 7.010 & 2.7246 & .006 \\
\hline & $\mathrm{N}$ & 25 & 3.976 & 1.3147 & \\
\hline \multirow{2}{*}{ S Protein } & $\mathrm{Y}$ & 10 & 6.450 & .9336 & .133 \\
\hline & $\mathrm{N}$ & 25 & 7.012 & .9888 & \\
\hline \multirow{2}{*}{$\mathrm{Hb}$} & $\mathrm{Y}$ & 10 & 8.050 & 3.4821 & .721 \\
\hline & $\mathrm{N}$ & 25 & 7.620 & 2.0700 & . \\
\hline \multirow{2}{*}{ TLC } & $\mathrm{Y}$ & 10 & 101350.00 & 150787.571 & .199 \\
\hline & $\mathrm{N}$ & 25 & 43412.00 & 103151.217 & \\
\hline \multirow{2}{*}{ Blast \% } & $\bar{Y}$ & 10 & 60.50 & 29.990 & .087 \\
\hline & $\mathrm{N}$ & 25 & 39.28 & 32.926 & \\
\hline
\end{tabular}

As $\mathrm{p}$ value is significant for age, baseline blood urea, serumcalcium and serum uric acid levels, so these factors are important predictors for development of tumor lysis syndrome and must be taken into consideration. Among 10 patients of acute leukemia who developed TLS, hyperuricemia, hyperkalemia, hyperphosphatemia and hypocalcemia were observed in equal number of cases. In Lymphoma group hyperuricemia and hyperkalemia were present in one third cases. All cases of TLS occurred during first cycle of chemotherapy, majority from day 0 to day 3 and cases of laboratory TLS recovered by day 6 after proper hydration and supportive management.

\section{Discussion}

In literature TLS was first reported almost 90 years ago by Bedrna and Polcák in chronic leukemia patients treated with irradiation in 1929 but its incidence remains ill-defined. The most commonly referenced percentages are from Hande and Garrow's 1993, who reported the incidence of tumourlysis syndrome identified through serial measurements of laboratory values to be $42 \%$, whereas the incidence of clinically significant TLS was only $6 \%$ in the same population. ${ }^{5}$

Wasim et al. ${ }^{6}$ studied 50 patients diagnosed with Haematological malignancies to determine the frequency of TLS and reported the incidence of $14 \%, 4 \%$, and $2 \%$ for acute leukemia, NHL, and chronic leukemia, respectively. This descriptive study was conducted at Liaquat National Postgraduate Medical Centre from October 2005 to April 2006 over a period of six months. Total 50 patients with diagnosed haematological malignancies were included in which $20 \%$ of the patients developed TLS (including both laboratory and clinical TLS) despite all measures of prevention. Among hematologic cancers, rates were highest for acute leukemia (26.0\% for LTLS, 
$16.4 \%$ for CTLS) and multiple myeloma (21.7\% for LTLS, $20.3 \%$ for CTLS). Rates of hyperuricemia by cancer type were approximately $63 \%$ for hematological malignancies.

In a multicenter cohort study ${ }^{7}$ of 153 high-risk patients with acute leukemia, aggressive NHL, and Burkitt leukemia/lymphoma, the overall incidence of TLS was found to be $30.7 \%$. This prospective multicentre cohort study included 153 consecutive patients with malignancies at high risk for TLS (median age 54 years (interquartile range, 38-66). Underlying malignancies were acute leukaemia (58\%), aggressive non-Hodgkin lymphoma (29.5\%), and Burkittleukaemia/ lymphoma (12.5\%). Laboratory TLS developed in $17(11.1 \%)$ patients and clinical TLS with acute kidney injury (AKI) in 30 (19.6\%) patients. After adjustment for confounders, admission phosphates level (odds ratio [OR] per mmol/l, 5.3; 95\% confidence interval $[95 \% \mathrm{CI}], 1.5-18.3)$, lactic dehydrogenase (OR per x normal, 1.1; 95\%CI, 1.005-1.25), and disseminated intravascular coagulation (OR, 4.1; 95\%CI, 1.4-12.3) were associated with clinical TLS; and TLS was associated with day-90 mortality (OR, 2.45; 95\%CI, 1.09-5.50; $\mathrm{P}=0.03)$. In this study, TLS occurred in $30.7 \%$ of high-risk patients. One third of all patients experienced AKI, for which TLS was an independent risk factor. TLS was associated with increased mortality, indicating a need for interventional studies aimed at decreasing early TLS-related deaths in this setting.Ahsan Ejaz et al. also reported $32 \%$ incidence of TLS in acute myeloid leukemia (AML) patients using retrospective analysis of 183 patients. ${ }^{8}$

In our study out of 100 patients 19 fulfilled the criteria for TLS.In acute leukemia group 10 (28.57\%) patients developed TLS of which 5 patients $(14.3 \%)$ had spontaneous tumor lysis syndrome, 4 patients (11.4\%) had laboratory tumor lysis syndrome and only one patient (2.9\%) had clinical tumor lysis syndrome. Among lymphoma group no patient developed spontaneous or clinical tumor lysis syndrome. Only laboratory tumor lysis syndrome was seen in
$9(13.8 \%)$ out of 65 patients of lymphoma. Overall one patient died because of TLS. It is indicated by the results of our study that in spite of available preventive measures TLS can occur and result in significant morbidity and mortality. That is why, it is important to recognize those patients who are at risk for the development of complications or death second ary to TLS.

\section{Conclusion}

Tumor lysis syndrome is a life-threatening complication of malignancy. Early identification of patients at risk and prevention is of crucial importance. It is seen commonly after chemotherapy which initiates massive cell death and lysis of cell contents. During treatment such patients should be observed and monitored closely. Uric acid, Creatinine, potassium, calcium and phosphate levels should be measured at the time of presentation and thereafter frequently in order to diagnose LTLS and prevent CTLS, which is potentially fatal. These biochemical abnormalities can be concurrently exacerbated by renal failure. It is recommended that prompt recognition and aggressive management of this life threatening complication by aggressive fluid and electrolyte management is essential to treat this condition which can occur in almost all haematological malignancies. Biochemical changes occurring should be corrected and monitored to prevent serious complications such as seizures, cardiac arrhythmias, renal failure and death. The present study upholds the prospects of prevalence of tumor lysis syndrome as described in literature but considering the small and study size and in the light of a few studies with contradictory results, further multicentric trials are indeed necess ary to establish its efficacy.

\section{References}

1. Cairo M, Coiffier B, Reiter A, Younes A. Recommendations forthe evaluation of risk and prophylaxis of tumour lysis syndrome (TLS) in adults and children with malignant diseases : An expert TLS panel 
consensus. British Journal Of

Haematology. 2010; 149(4):578-86.

2. Krishnan G, D'silva K, Al-Janadi A. Cetuximab related tumourlysis syndrome in metastatic colon carcinoma. Journal of clinical oncology. 2008;26(14):2406-8.

3. Joshita S, Yoshizava K, Sano K, Kobayashi S, Sekiguchi T, Morita S et al. A patient with advanced hepatocellular carcinoma treated with sorafenib Tosylate Showed massive tumor lysis with avoidance of tumor lysis syndrome. In ternal medicine. 2010;49(11):991-4.

4. Cairo M, Bishop M. Tumor lysis Syndrome: New therapeutic strategies and classification. British Journal Of Haematology. 2004; 127(1):3-11.

5. Hande KR, Garrow JC. Acute tumor lysis syndrome with high grade Non-Hodgkin's Lymphoma. Am J Med. 1993;94(2):133-9

6. Wasim F, Khaskheli A. M., Siddiqui A. A., Tariq O., Ansari M. A. Tumor lysis syndrome in haematological malignancies. Journal of the Liaquat University of Mental Health and Sciences. 2012;11(2):84-9

7. Darmon M, Vincent F et.al. Groupe. A prospective multicentricstudy from the Groupe de Rechercheen Reanimation Respiratoireet Onco- Hematologique. Br J Haematol.2013 Aug; 162(4): 489-97

8. Ejaz AA, Pourafshar N, Mohandas R et al. Uric acid and prediction models of tumor lysis Syndrome in AML. PLoS ONE. 2015;10(3) : e0119497. 